\title{
Existence of Solutions for Fractional Impulsive Integrodifferential Equations in Banach Spaces
}

\author{
Haide Gou and Baolin Li \\ College of Mathematics and Statistics, Northwest Normal University, Lanzhou 730070, China \\ Correspondence should be addressed to Baolin Li; ghdzxh@163.com
}

Received 14 September 2016; Accepted 1 November 2016

Academic Editor: Yuji Liu

Copyright ( 92016 H. Gou and B. Li. This is an open access article distributed under the Creative Commons Attribution License, which permits unrestricted use, distribution, and reproduction in any medium, provided the original work is properly cited.

\begin{abstract}
We investigate the existence of solutions for a class of impulsive fractional evolution equations with nonlocal conditions in Banach space by using some fixed point theorems combined with the technique of measure of noncompactness. Our results improve and generalize some known results corresponding to those obtained by others. Finally, two applications are given to illustrate that our results are valuable.
\end{abstract}

\section{Introduction}

Fractional differential equations arise in many engineering and scientific disciplines as the mathematical modelling of systems and processes in the fields of physics, chemistry, aerodynamics, electrodynamics of complex medium, and polymer rheology and have been emerging as an important area of investigation in the last few decades; see [1-4]. However, the theory of impulsive fractional evolution equations was still in the initial stages and many aspects of this theory need to be explored.

The theory of impulsive differential equations is a new and important branch of differential equation theory, which has an extensive physical, population dynamics, ecology, chemical, biological systems, and engineering background. Therefore, it has been an object of intensive investigation in recent years; some basic results on impulsive differential equations have been obtained and applications to different areas have been considered by many authors; see [4-8].

The study of nonlocal Cauchy problem for abstract evolution differential equations has been initiated by Byszewski [9]. The existence of solutions for fractional abstract differential equations with nonlocal initial condition was recently investigated by N'Guérékata [10] and Balachandran and Park [11]. In [12], Balachandran et al. were concerned with the existence of solutions of first-order nonlinear impulsive fractional integrodifferential equations in Banach spaces:

$$
\begin{aligned}
{ }^{c} D_{t}^{\alpha} u(t)= & A u(t) \\
& +f\left(t, u(t), \int_{0}^{t} h(t, s, u(s)) d s\right), \\
\left.\Delta u\right|_{t=t_{k}} & =I_{k}\left(u\left(t_{k}^{-}\right)\right), \quad k=1, \ldots, m, \\
u(0)+g(u)= & u_{0} ;
\end{aligned}
$$

the results are obtained by using fixed point principles.

Shu and Wang [4] studied the existence of mild solutions for fractional differential equation with nonlocal conditions in a Banach space $E$ :

$$
\begin{aligned}
{ }^{c} D_{t}^{\alpha} u(t)= & A u(t)+f(t, u(t)) \\
& +\int_{0}^{t} q(t-s) g(s, u(s)) d s,
\end{aligned}
$$

$$
t \in[0, T],
$$

$$
\begin{aligned}
& u(0)+m(u)=u_{0} \in E, \\
& u^{\prime}(0)+n(u)=u_{1} \in E .
\end{aligned}
$$


By using the contraction mapping principle and Krasnoselskii's fixed point theorem, they obtained the existence of solutions for the equation.

In [13], Gou and Li investigated local and global existence of mild solution for an impulsive fractional functional integrodifferential equation with noncompact semigroup in Banach spaces $E$ :

$$
\begin{aligned}
{ }^{c} D_{t}^{\alpha} u(t)+A u(t)= & f(t, u(t)) \\
& +\int_{0}^{t} q(t-s) g(s, u(s)) d s, \\
t \geq 0, t \neq t_{k}, & t, \ldots, m, \\
\left.\Delta u\right|_{t=t_{k}}= & I_{k}\left(u\left(t_{k}^{-}\right)\right), \quad k=1, \ldots, \\
u(0) & =u_{0} \in E,
\end{aligned}
$$

and they establish a general framework to find the mild solutions for impulsive fractional integrodifferential equations, which will provide an effective way to deal with such problems.

Motivated by this consideration, we investigate the existence of solutions for a class of impulsive fractional evolution equations with nonlocal conditions in Banach space $E$ :

$$
\begin{aligned}
{ }^{c} D_{t}^{\alpha} u(t)+A u(t) & =f(t, u(t),(F u)(t),(G u)(t)), \\
t \in J, t \neq t_{k}, & \\
\left.\Delta u\right|_{t=t_{k}} & =I_{k}\left(u\left(t_{k}^{-}\right)\right), \quad k=1,2, \ldots, m, \\
u(0) & =g(u)+u_{0},
\end{aligned}
$$

by using some fixed point theorems combined with the technique of measure of noncompactness, where ${ }^{c} D_{t}^{\alpha}$ is the Caputo fractional derivative of order $\alpha \in(1,2], A: D(A) \subset$ $E \rightarrow E$ is a closed linear operator, and $-A$ generates a $C_{0^{-}}$ semigroup $T(t)(t \geq 0)$ in $E ; f: J \times E \times E \times E \rightarrow E, J=[0, a]$, where $a>0$ is a constant, $0=t_{0}<t_{1}<t_{2}<\cdots<t_{m}<t_{m+1}=$ $a ; I_{k}: E \rightarrow E$ is an impulsive function, and $k=1,2, \ldots, m$; and $x_{0} \in E, p_{1}, p_{2}$ is an $E$-valued function to be given later and

$$
\begin{array}{ll}
(F u)(t)=\int_{0}^{t} k(t, s) p_{1}(s, u(s)) d s, & k \in C\left(D, R^{+}\right), \\
(G u)(t)=\int_{0}^{a} h(t, s) p_{2}(s, u(s)) d s, & h \in C\left(D_{0}, R^{+}\right),
\end{array}
$$

$D=\left\{(t, s) \in R^{2}: 0 \leq s \leq t \leq a\right\}, D_{0}=\left\{(t, s) \in R^{2}: 0 \leq\right.$ $t, s \leq a\}, p_{i} \in C(J \times E, E), i=1,2$, and $\left.\Delta u\right|_{t=t_{k}}$ denotes the jump of $u(t)$ at $t=t_{k}$; that is, $\left.\Delta u\right|_{t=t_{k}}=u\left(t_{k}^{+}\right)-u\left(t_{k}^{-}\right), u\left(t_{k}^{+}\right)$ and $u\left(t_{k}^{-}\right)$represent the right and left limits of $u(t)$ at $t=t_{k}$, respectively.

The paper is organized as follows. In Section 2, we recall some concepts and facts about the Kuratowski measure of noncompactness and some fixed point theorems. In Section 3, we obtain the existence solutions of problem (4). In Section 4, we give two examples to illustrate our results.

\section{Preliminaries}

In this section, we briefly recall some definitions and the fixed point theorems which will be used in the sequel. Throughout this paper, let $E$ be a Banach space; we assume that $A$ : $D(A) \quad \subset \quad E \rightarrow E$ is a closed linear operator and $-A$ generates a uniformly bounded $C_{0}$-semigroup $T(t)(t>0)$ on a Banach space $(E,\|\cdot\|)$ and $M=\sup _{t \in[0,+\infty)}\|T(t)\|_{B(E)}$, where $B(E)$ stands for the Banach space of all linear and bounded operators in $E$.

$C(J, E)$ denote the Banach space of all continuous $E$-value functions on interval $J$ and $P C(J, E)=\{u: J \rightarrow E$ : $u \in C\left(\left(t_{k-1}, t_{k}\right], E\right), k=1,2, \ldots, m$, and there exist $u\left(t_{k}^{-}\right)$and $u\left(t_{k}^{+}\right), k=1,2, \ldots, m$ with $\left.u\left(t_{k}^{-}\right)=u\left(t_{k}\right)\right\}$. Obviously, $P C(J, E)$ is a Banach space with the supnorm $\|u\|=\sup _{t \in J}\|u(t)\|$.

Definition 1. The fractional integral of order $\alpha>0$ with the lower limit zero for a function $u$ is defined as

$$
I_{t}^{\alpha} u(t)=\frac{1}{\Gamma(\alpha)} \int_{0}^{t}(t-s)^{\alpha-1} u(s) d s, \quad t>0
$$

where $\Gamma(\cdot)$ is the Gamma function.

Definition 2. The Caputo fractional derivative of order $\alpha>0$ with the lower limit zero for a function $u$ is defined as

$$
\begin{array}{r}
{ }^{c} D_{t}^{\alpha} u(t)=\frac{1}{\Gamma(n-\alpha)} \int_{0}^{t}(t-s)^{n-\alpha-1} u^{(n)}(s) d s, \\
t>0,0 \leq n-1<\alpha<n,
\end{array}
$$

where the function $u(t)$ has absolutely continuous derivatives up to order $n-1$.

Remark 3. If $u$ is an abstract function with values in $E$, then the integrals which appear in Definitions 1 and 2 are taken in Bochner's sense.

Definition 4. By a mild solution of the initial value problem

$$
\begin{aligned}
{ }^{c} D_{t}^{\alpha} u(t)+A u(t) & =f(t, u(t)), \quad t \geq 0, \\
u(0) & =u_{0},
\end{aligned}
$$

on $[0, \infty)$, we mean that a continuous function $u$ defined from $[0, \infty)$ into $E$ satisfying

$$
\begin{aligned}
u(t)= & \mathscr{T}_{\alpha}(t) u_{0} \\
& +\int_{0}^{t}(t-s)^{\alpha-1} \mathcal{S}_{\alpha}(t-s) f(s, u(s)) d s, \\
& \quad t \in[0, \infty),
\end{aligned}
$$


where

$$
\begin{aligned}
& \mathscr{T}_{\alpha}(t)=\int_{0}^{\infty} \theta_{\alpha}(s) T\left(t^{\alpha} s\right) d s, \\
& \mathcal{S}_{\alpha}(t)=\alpha \int_{0}^{\infty} s \theta_{\alpha}(s) T\left(t^{\alpha} s\right) d s, \\
& \theta_{\alpha}(s)=\frac{1}{\pi \alpha} \sum_{n=1}^{\infty}(-s)^{n-1} \frac{\Gamma(n \alpha+1)}{n !} \sin (n \pi \alpha), \\
& s \in(0, \infty)
\end{aligned}
$$

are the functions of Wright type defined on $(0, \infty)$ which satisfies

$$
\begin{gathered}
\int_{0}^{\infty} \theta_{\alpha}(s) d s=1, \quad \theta_{\alpha}(s) \geq 0, s \in(0, \infty), \\
\int_{0}^{\infty} s^{v} \theta_{\alpha}(s) d s=\frac{\Gamma(1+v)}{\Gamma(1+\alpha v)}, \quad v \in[0,1] .
\end{gathered}
$$

The following lemma can be found in $[14,15]$.

Lemma 5. The operators $\mathscr{T}_{\alpha}(t)$ and $\mathcal{S}_{\alpha}(t)(t \geq 0)$ have the following properties:

(i) For any fixed $t \geq 0, \mathscr{T}_{\alpha}(t)$ and $\mathcal{S}_{\alpha}(t)$ are linear and bounded operators; that is, for any $u \in E$,

$$
\begin{aligned}
\left\|\mathscr{T}_{\alpha}(t) u\right\| & \leq M\|u\|, \\
\left\|\mathcal{S}_{\alpha}(t) u\right\| & \leq \frac{\alpha M}{\Gamma(\alpha+1)}\|u\|=\frac{M}{\Gamma(\alpha)}\|u\| .
\end{aligned}
$$

(ii) For every $u \in E, t \rightarrow \mathscr{T}_{\alpha}(t) u$ and $t \rightarrow \mathcal{S}_{\alpha}(t) u$ are continuous functions from $[0, \infty)$ into $E$.

(iii) The operators $\mathscr{T}_{\alpha}(t)(t \geq 0)$ and $\mathcal{S}_{\alpha}(t)(t \geq 0)$ are strongly continuous, which means that, for $\forall u \in E$ and $0 \leq t^{\prime}<t^{\prime \prime} \leq a$, one has

$$
\begin{gathered}
\left\|\mathscr{T}_{\alpha}\left(t^{\prime \prime}\right) u-\mathscr{T}_{\alpha}\left(t^{\prime}\right) u\right\| \longrightarrow 0, \\
\left\|\mathcal{S}_{\alpha}\left(t^{\prime \prime}\right) u-\mathcal{S}_{\alpha}\left(t^{\prime}\right) u\right\| \longrightarrow 0,
\end{gathered}
$$

$$
\text { as } t^{\prime \prime}-t^{\prime} \longrightarrow 0 \text {. }
$$

(iv) If the semigroup $T(t)$ is continuous by operator norm for every $t>0$, then $\mathscr{T}_{\alpha}(t)$ and $\mathcal{S}_{\alpha}(t)$ are continuous in $(0,+\infty)$ by the operator norm.

Let $\beta(\cdot)$ denote the Kuratowski measure of noncompactness of the bounded set. For the details of the definition and properties of the measure of noncompactness, see [6]. results.

The following lemmas are to be used in proving our main

Lemma 6 (see [16]). Let $E$ be a Banach space, and let $D \subset C(J, E)$ be equicontinuous and bounded; then $\beta(D(t))$ is continuous on $J$, and $\beta(D)=\max _{t \in J} \beta(D(t))$.

Lemma 7 (see [7]). Let $D \subset E$ be bounded. Then there exists a countable set $D_{0} \subset D$, such that $\beta(D) \leq 2 \beta\left(D_{0}\right)$.
Lemma 8 (see [17]). Let $E$ be a Banach space, and let $D=$ $\left\{u_{n}\right\} \subset C(I, X)$ be a bounded and countable set. Then $\beta(D(t))$ is the Lebesgue integral on $X$, and

$$
\beta\left(\left\{\int_{I} u_{n}(t) d t \mid n \in \mathbb{N}\right\}\right) \leq 2 \int_{I} \beta(D(t)) d t
$$

In the following, we introduce the definition of $(\gamma, \psi, p)$ contractive mapping.

Definition 9 (see [18]). Let $(E, d)$ be a metric space with $w$ distance $p$ and $f: E \rightarrow E$ a given mapping. We say that $f$ is an $(\gamma, \psi, p)$-contractive mapping if there exist two functions $\gamma: E \times E \rightarrow[0, \infty)$ and $\psi \in \Psi$ such that

$$
\gamma(x, y) p(f x, f y) \leq \psi(p(x, y))
$$

for all $x, y \in E$.

We will show some fixed point theorems about condensing operator and $(\gamma, \psi, p)$-contractive mapping, which play a key role in the proof of our main results.

Lemma 10 (see [19]). Let E be a Banach space. Assume that $D \subset E$ is a bounded closed and convex set on $E, Q: D \rightarrow D$ is condensing. Then $Q$ has at least one fixed point in $D$.

Lemma 11 (see [18]). Let $p$ be a $w$ distance on a complete metric space $(E, d)$ and let $f: E \rightarrow E$ be an $(\gamma, \psi, p)$ contractive mapping. Suppose that the following conditions hold:

(i) $f$ is an $\gamma$-admissible mapping;

(ii) there exists a point $x_{0} \in E$ such that $\gamma\left(x_{0}, f x_{0}\right) \geq 1$;

(iii) either $f$ is continuous or, for any sequence $\left\{x_{n}\right\}$ in $E$, if $\gamma\left(x_{n}, x_{n+1}\right) \geq 1$ for all $n \in \mathbb{N}$ and $x_{n} \rightarrow x \in E$ as $n \rightarrow \infty$, then $\gamma\left(x_{n}, x\right) \geq 1$ for all $n \in \mathbb{N}$. Then there exists a point $u \in E$ such that $f u=u$. Moreover, if $\gamma(u, u) \geq 1$, then $p(u, u)=0$.

\section{Main Results}

In this section, we will establish the existence theorems of solutions for the nonlocal problem (4). For convenience, we give some notations.

For $B \subset C(J, E)$, let $B(t)=\{u(t): u \in B\}$ and denote $B_{R}=\{u \in E:\|u\| \leq R\}$. Let $k_{0}=\max \{k(t, s):(t, s) \in D\}$, $h_{0}=\max \left\{h(t, s):(t, s) \in D_{0}\right\}$. First of all, let us start by defining what we mean by a solution of problem (4).

Definition 12. A function $u \in P C(J, E)$ is said to be a mild solution of problem (4) if $u$ satisfies the equation

$$
\begin{gathered}
u(t)=\mathscr{T}_{\alpha}(t)\left(g(u)+u_{0}\right)+\sum_{0<t_{k}<t} \int_{t_{k-1}}^{t_{k}}\left(t_{k}-s\right)^{\alpha-1} \\
\cdot \mathcal{S}_{\alpha}(t-s) f(s, u(s),(F u)(s),(G u)(s)) d s
\end{gathered}
$$




$$
\begin{aligned}
& +\int_{t_{k}}^{t}(t-s)^{\alpha-1} \mathcal{S}_{\alpha}(t-s) f(s, u(s),(F u)(s),(G u) \\
& \cdot(s)) d s+\sum_{0<t_{k}<t} \mathscr{T}_{\alpha}\left(t-t_{k}\right) I_{k}\left(u\left(t_{k}^{-}\right)\right) .
\end{aligned}
$$

To prove our main results, we state the following basic assumptions of this paper.

(H1) There exists a positive constant $K_{i},(i=1,2,3)$ such that

$$
\begin{aligned}
& \| f(t, u(t),(F u)(t),(G u)(t)) \\
& \quad-f(t, v(t),(F v)(t),(G v)(t))\left\|\leq K_{1}\right\| u-v \| \\
& \quad+K_{2}\|F u-F v\|+K_{3}\|G u-G v\|
\end{aligned}
$$

and for $p_{i} \in C(J \times E, E), t \in J, u, v \in B_{R}$, there exists a constant $b_{i}, c_{i}>0$ such that

$$
\begin{gathered}
\left\|p_{i}(t, u)-p_{i}(t, v)\right\| \leq b_{i}\|u-v\|, \\
\left\|p_{i}(t, u)\right\| \leq c_{i}\|u\|,
\end{gathered}
$$

$$
i=1,2 \text {. }
$$

(H2) For any $R>0$, there exist a Lebesgue-integrable function $M_{R}: I \rightarrow R^{+}$and nondecreasing continuous function $\Omega:[0, \infty) \rightarrow(0, \infty)$ such that

$$
\|f(t, x, y, z)\| \leq M_{R}(t) \Omega(R)
$$

for all $t \in I,(x, y, z) \in B_{R} \times B_{R} \times B_{R}$.

(H3) $g: E \rightarrow E$ is a continuous and compact mapping; furthermore, there exists positive number $N_{1}, N_{2}$ such that $\|g(u)-g(v)\| \leq N_{1}$ and $\|g(u)\| \leq N_{2}$ for any $u, v \in B_{R}$.

(H4) The functions $I_{k}: E \rightarrow E$ are continuous and there exists a constant $\mu>0$ and $\rho>0$ such that

$$
\begin{aligned}
&\left\|I_{k}(t, u)-I_{k}(t, v)\right\| \leq \mu\|u-v\|, \\
&\left\|I_{k}(u)\right\| \leq \rho\|u\|, \\
& \forall u, v \in B_{R}, k=1,2, \ldots, m .
\end{aligned}
$$

(H5) There exist constants $L_{i}>0,(i=1,2,3)$ such that for any bounded and equicontinuous sets $D_{i} \subset$ $C(J, E),(i=1,2,3)$ and $t \in J$,

$$
\begin{aligned}
& \beta\left(f\left(t, D_{1}, D_{2}, D_{3}\right)\right) \leq \sum_{i=1}^{3} L_{i} \beta\left(D_{i}\right), \\
& \beta\left(k(t, s) p_{1}\left(s, D_{4}\right)\right) \leq L_{4} \beta\left(D_{4}\right), \\
& \beta\left(h(t, s) p_{2}\left(s, D_{5}\right)\right) \leq L_{5} \beta\left(D_{5}\right) .
\end{aligned}
$$

Lemma 13 (see [13]). Let $f, g: J \times E \rightarrow E$ be a continuous function and let $-A$ be the generator of a $C_{0}$-semigroup $(T(t))(t \geq 0)$. If $u \in P C(J, E)$ is a mild solution of (4) in the sense of Definition 12, then for any $t \in\left(t_{k-1}, t_{k}\right], k=1, \ldots, m$,

$$
\begin{aligned}
& u(t)=\mathscr{T}_{\alpha}(t)\left(g(u)+u_{0}\right)+\sum_{0<t_{k}<t} \int_{t_{k-1}}^{t_{k}}\left(t_{k}-s\right)^{\alpha-1} \\
& \cdot \mathcal{S}_{\alpha}(t-s) f(s, u(s),(F u)(s),(G u)(s)) d s \\
& +\int_{t_{k}}^{t}(t-s)^{\alpha-1} \mathcal{S}_{\alpha}(t-s) f(s, u(s),(F u)(s),(G u) \\
& \cdot(s)) d s+\sum_{0<t_{k}<t} \mathscr{T}_{\alpha}\left(t-t_{k}\right) I_{k}\left(u\left(t_{k}^{-}\right)\right)
\end{aligned}
$$

is a solution of (4). In other words $u(t)$ is a mild solution of (4).

Theorem 14. Let $E$ be a Banach space, let $A: D(A) \subset E \rightarrow E$ be a closed linear operator, and -A generates an equicontinuous $C_{0}$-semigroup $T(t)(t \geq 0)$ of uniformly bounded operators in E. Suppose that the conditions (H1)-(H5) are satisfied. Then for every $u_{0} \in P C(J, E)$ there exists a $\tau_{1}=\tau_{1}\left(u_{0}\right), 0<\tau_{1}<$ a such that problem (4) has a solution $u \in P C\left(\left[0, \tau_{1}\right], E\right)$.

Proof. Since we are interested here only in local solutions, we may assume that $a<\infty$. By using our assumption (H1)-(H4), let $t^{\prime}>0, R=M\left(2\left\|u_{0}\right\|+1\right)>0$ be such that $B_{R}\left(u_{0}\right)=\{u$ : $\left.\left\|u-u_{0}\right\| \leq R\right\}$, for $0 \leq t \leq t^{\prime}$ and $u \in B_{R}\left(u_{0}\right)$, and let us choose

$$
\begin{aligned}
\tau_{1}= & \min \left\{t^{\prime}, a,\right. \\
& {\left[\frac{\Gamma(\alpha+1)\left(\left\|x_{0}\right\|+1-N_{2}-m \rho_{1}\right)}{(m+1)\left\|M_{R}\right\| \Omega\left(M\left(2\left\|u_{0}\right\|+1\right)\right)}\right]^{1 / \alpha}, } \\
& {\left.\left[\frac{\Gamma(\alpha+1)}{2 M(m+1)\left(L_{1}+a k_{0} L_{2} L_{4}+a h_{0} L_{3} L_{5}\right)}\right]^{1 / \alpha}\right\} . }
\end{aligned}
$$

Set $\Omega=\left\{u \in P C\left(\left[0, \tau_{1}\right], E\right):\|u(t)\| \leq R, t \in\left[0, \tau_{1}\right]\right\}$; then $\Omega$ is a closed ball in $P C\left(\left[0, \tau_{1}\right], E\right)$ with center $\theta$ and radius $R$. Consider the operator $Q: \Omega \rightarrow P C\left(\left[0, \tau_{1}\right], E\right)$ defined by

$$
\begin{aligned}
& (Q u)(t)=\mathscr{T}_{\alpha}(t)\left(g(u)+u_{0}\right)+\sum_{0<t_{k}<t} \int_{t_{k-1}}^{t_{k}}\left(t_{k}-s\right)^{\alpha-1} \\
& \cdot \mathcal{S}_{\alpha}(t-s) f(s, u(s),(F u)(s),(G u)(s)) d s \\
& +\int_{t_{k}}^{t}(t-s)^{\alpha-1} \mathcal{S}_{\alpha}(t-s) f(s, u(s),(F u)(s),(G u) \\
& \cdot(s)) d s+\sum_{0<t_{k}<t} \mathscr{T}_{\alpha}\left(t-t_{k}\right) I_{k}\left(u\left(t_{k}^{-}\right)\right) .
\end{aligned}
$$

It is easy to see that the fixed points of $Q$ are the solutions of the nonlocal problem (4); we shall prove that $Q$ has a fixed 
point by using Lemma 10 . For any $u \in \Omega$ and $t \in\left[0, \tau_{1}\right]$, by Lemma 5(i), we have

$$
\begin{aligned}
\|(F u)(s)\| & =\left\|\int_{0}^{t} k(t, s) p_{1}(s, u(s)) d s\right\| \\
& \leq k_{0} \int_{0}^{a}\left\|p_{1}(s, u(s))\right\| d s \leq R c_{1} k_{0} \leq R,
\end{aligned}
$$

where $c_{1}=\left(k_{0}\right)^{-1}$. So $T x \in \Omega$. Similarly, we prove $G u \in \Omega$.

And by (19), we have

$$
\begin{aligned}
& \|(Q u)(t)\| \\
& \leq\left\|\mathcal{T}_{\alpha}(t)\left(g(u)+u_{0}\right)\right\| \\
& \quad+\frac{M\left\|M_{R}\right\| \Omega(R)}{\Gamma(\alpha)} \sum_{0<t_{k}<t} \int_{t_{k-1}}^{t_{k}}\left(t_{k}-s\right)^{\alpha-1} d s \\
& \quad+\frac{M\left\|M_{R}\right\| \Omega(R)}{\Gamma(\alpha)} \int_{t_{k}}^{t}(t-s)^{\alpha-1} d s \\
& \quad+M \sum_{0<t_{k}<t} I_{k}\left(u\left(t_{k}^{-}\right)\right) \\
& \leq M\left(N_{2}+\left\|u_{0}\right\|\right)+\frac{M\left\|M_{R}\right\| \Omega(R)(m+1) \tau_{1}^{\alpha}}{\Gamma(\alpha+1)} \\
& +M m \rho \leq R .
\end{aligned}
$$

Therefore, $Q u \in \Omega$. Now we show that $Q$ is continuous from $\Omega$ into $\Omega$. To show this, we first observe that since $f$ is continuous in $[0, a] \times E$, it follows that for any $\epsilon>0$ and for a fixed $u \in B_{R}\left(u_{0}\right)$ there exists $\delta_{1}(u), \delta_{2}(u)>0$ such that for any $v \in B_{R}\left(u_{0}\right)$ and let $\delta(u)=\min \left\{\delta_{1}(u), \delta_{2}(u)\right\}$. Then for any $v \in \Omega,\|u-v\|<\delta(u)$ and choose

$$
\begin{aligned}
& \left(\frac{M(m+1)\left(K_{1}+K_{2} a k_{0} b_{1}+K_{3} a h_{0} b_{2}\right) \tau_{1}^{\alpha}}{\Gamma(\alpha+1)}\right. \\
& \left.+M\left(m \mu+N_{1}\right)\right)<\frac{\epsilon}{\delta(u)} .
\end{aligned}
$$

Then we have

$$
\begin{aligned}
& \|(Q u)(t)-(Q v)(t)\| \leq \mathscr{T}_{\alpha}(t)\|(g(u)-g(v))\| \\
& +\sum_{0<t_{k}<t} \int_{t_{k-1}}^{t_{k}}\left(t_{k}-s\right)^{\alpha-1}\left\|\mathcal{S}_{\alpha}(t-s)\right\| \\
& \cdot \| f(s, u(s),(F u)(s),(G u)(s)) \\
& -f(s, v(s),(F v)(s),(G v)(s)) \| d s+\int_{t_{k}}^{t}(t \\
& -s)^{\alpha-1}\left\|\mathcal{S}_{\alpha}(t-s)\right\| \\
& \cdot \| f(s, u(s),(F u)(s),(G u)(s))
\end{aligned}
$$

$$
\begin{aligned}
& -f(s, v(s),(F v)(s),(G v)(s)) \| d s \\
& +\sum_{0<t_{k}<t}\left\|\mathscr{T}_{\alpha}\left(t-t_{k}\right)\right\|\left\|I_{k}\left(u\left(t_{k}^{-}\right)\right)-I_{k}\left(v\left(t_{k}^{-}\right)\right)\right\| \\
& \leq\left(\frac{M(m+1)\left(K_{1}+K_{2} a k_{0} b_{1}+K_{3} a h_{0} b_{2}\right) \tau_{1}^{\alpha}}{\Gamma(\alpha+1)}\right. \\
& \left.+M\left(m \mu+N_{1}\right)\right)\|u-v\| \leq \epsilon .
\end{aligned}
$$

Thus, we proved that $Q: \Omega \rightarrow \Omega$ is a continuous operator.

Now, we demonstrate that the operator $Q: \Omega \rightarrow \Omega$ is equicontinuous. For any $u \in \Omega$ and $0 \leq t_{1}<t_{2} \leq \tau_{1}$, we get that

$$
\begin{aligned}
& \left\|(Q u)\left(t_{2}\right)-(Q u)\left(t_{1}\right)\right\|=\mathscr{T}_{\alpha}\left(t_{2}\right)\left(g(u)+u_{0}\right) \\
& -\mathscr{T}_{\alpha}\left(t_{1}\right)\left(g(u)+u_{0}\right) \\
& +\sum_{0<t_{k}<t} \int_{t_{k-1}}^{t_{k}}\left(t_{k}-s\right)^{\alpha-1}\left(\mathcal{S}_{\alpha}\left(t_{2}-s\right)-\mathcal{S}_{\alpha}\left(t_{1}-s\right)\right) \\
& \times f(s, u(s),(F u)(s),(G u)(s)) d s+\int_{t_{1}}^{t_{2}}\left(t_{2}\right. \\
& -s)^{\alpha-1} \mathcal{S}_{\alpha}\left(t_{2}-s\right) \\
& \cdot f(s, u(s),(F u)(s),(G u)(s)) d s \\
& +\int_{t_{k}}^{t_{1}}\left(\left(t_{2}-s\right)^{\alpha-1}-\left(t_{1}-s\right)^{\alpha-1}\right) \mathcal{S}_{\alpha}\left(t_{2}-s\right) \\
& \cdot f(s, u(s),(F u)(s),(G u)(s)) d s+\int_{t_{k}}^{t_{1}}\left(t_{1}\right. \\
& +s)^{\alpha-1}\left(\mathcal{S}_{\alpha}\left(t_{2}-s\right)-\mathcal{S}_{\alpha}\left(t_{1}-s\right)\right) \\
& +f(s, u(s),(F u)(s),(G u)(s)) d s \\
& +\sum_{0<t_{k}<t}\left(\mathscr{T}_{\alpha}\left(t_{2}-t_{k}\right)-\mathscr{T}_{\alpha}\left(t_{1}-t_{k}\right)\right) \times I_{k}\left(u\left(t_{k}^{-}\right)\right) \\
& +I_{1}+I_{2}+I_{3}+I_{4}+I_{5}+I_{6},
\end{aligned}
$$

where

$$
\begin{aligned}
I_{1} & =\mathscr{T}_{\alpha}\left(t_{2}\right)\left(g(u)+u_{0}\right)-\mathscr{T}_{\alpha}\left(t_{1}\right)\left(g(u)+u_{0}\right), \\
I_{2} & =\sum_{0<t_{k}<t} \int_{t_{k-1}}^{t_{k}}\left(t_{k}-s\right)^{\alpha-1}\left(\mathcal{S}_{\alpha}\left(t_{2}-s\right)-\mathcal{S}_{\alpha}\left(t_{1}-s\right)\right) \\
& \times f(s, u(s),(F u)(s),(G u)(s)) d s, \\
I_{3} & =\int_{t_{1}}^{t_{2}}\left(t_{2}-s\right)^{\alpha-1} \mathcal{S}_{\alpha}\left(t_{2}-s\right) f(s, u(s),(F u)(s),(G u)
\end{aligned}
$$

$\cdot(s)) d s$ 


$$
\begin{aligned}
I_{4} & =\int_{t_{k}}^{t_{1}}\left(\left(t_{2}-s\right)^{\alpha-1}-\left(t_{1}-s\right)^{\alpha-1}\right) \mathcal{S}_{\alpha}\left(t_{2}-s\right) \\
& \cdot f(s, u(s),(F u)(s),(G u)(s)) d s, \\
I_{5} & =\int_{t_{k}}^{t_{1}}\left(t_{1}-s\right)^{\alpha-1}\left(\mathcal{S}_{\alpha}\left(t_{2}-s\right)-\mathcal{S}_{\alpha}\left(t_{1}-s\right)\right) \\
& \cdot f(s, u(s),(F u)(s),(G u)(s)) d s, \\
I_{6}= & \sum_{0<t_{k}<t}\left(\mathscr{T}_{\alpha}\left(t_{2}-t_{k}\right)-\mathscr{T}_{\alpha}\left(t_{1}-t_{k}\right)\right) \times I_{k}\left(u\left(t_{k}^{-}\right)\right) .
\end{aligned}
$$

Here we calculate

$$
\left\|(Q u)\left(t_{2}\right)-(Q u)\left(t_{1}\right)\right\| \leq \sum_{i=1}^{6}\left\|I_{i}\right\| .
$$

Therefore, we inspect that $\left\|I_{i}\right\|$ tend to 0 , when $t_{2}-t_{1} \rightarrow 0$, $i=1,2, \ldots, 6$.

For $I_{1}$, by Lemma 5(iii), $\left\|I_{1}\right\| \rightarrow 0$ as $t_{2}-t_{1} \rightarrow 0$.

For $I_{2}$, by Lemma 5(iii), we have

$$
\begin{aligned}
& \left\|I_{2}\right\| \\
& \quad \leq \sum_{0<t_{k}<t} \int_{t_{k-1}}^{t_{k}}\left(t_{k}-s\right)^{\alpha-1}\left\|\delta_{\alpha}\left(t_{2}-s\right)-\delta_{\alpha}\left(t_{1}-s\right)\right\| \\
& \cdot\left\|M_{R}\right\| \Omega(R) d s \rightarrow 0, \quad t_{2}-t_{1} \longrightarrow 0 .
\end{aligned}
$$

For $I_{3}$, by Lemma 5(i), we have

$$
\begin{aligned}
\left\|I_{3}\right\| \leq \frac{M\left\|M_{R}\right\| \Omega(R)}{\Gamma(\alpha)} \int_{t_{1}}^{t_{2}}\left(t_{2}-s\right)^{\alpha-1} d s & \\
\leq \frac{M\left\|M_{R}\right\| \Omega(R)}{\Gamma(\alpha+1)}\left(t_{2}-t_{1}\right)^{\alpha} \longrightarrow 0, & \\
& t_{2}-t_{1} \longrightarrow 0 .
\end{aligned}
$$

For $I_{4}$, by Lemma 5(i), we have

$$
\begin{aligned}
& \left\|I_{4}\right\| \leq \frac{M\left\|M_{R}\right\| \Omega(R)}{\Gamma(\alpha)} \\
& \cdot \int_{t_{k}}^{t_{1}}\left(\left(t_{2}-s\right)^{\alpha-1}-\left(t_{1}-s\right)^{\alpha-1}\right) d s
\end{aligned}
$$

$$
\begin{aligned}
& \leq \frac{M\left\|M_{R}\right\| \Omega(R)}{\Gamma(\alpha+1)}\left(\left(t_{1}-t_{2}\right)^{\alpha}-\left(t_{k}-t_{2}\right)^{\alpha}\right. \\
& \left.+\left(t_{k}-t_{1}\right)^{\alpha}\right) \longrightarrow 0, \quad t_{2}-t_{1} \longrightarrow 0 .
\end{aligned}
$$

For $I_{5}$, by Lemma 5(iii), we have

$$
\begin{gathered}
\left\|I_{5}\right\| \leq \int_{t_{k}}^{t_{1}}\left(t_{1}-s\right)^{\alpha-1}\left\|\mathcal{S}_{\alpha}\left(t_{2}-s\right)-\mathcal{S}_{\alpha}\left(t_{1}-s\right)\right\| \\
\cdot\left\|M_{R}\right\| \Omega(R) d s \longrightarrow 0, \quad t_{2}-t_{1} \longrightarrow 0 .
\end{gathered}
$$

For $I_{6}$, by Lemma 5(iii), we have

$$
\begin{aligned}
&\left\|I_{6}\right\| \leq m \rho\left\|\mathscr{T}_{\alpha}\left(t_{2}-t_{k}\right)-\mathscr{T}_{\alpha}\left(t_{1}-t_{k}\right)\right\| \longrightarrow 0, \\
& t_{2}-t_{1} \longrightarrow 0 .
\end{aligned}
$$

In conclusion, $\left\|(Q u)\left(t_{2}\right)-(Q u)\left(t_{1}\right)\right\|$ tends to 0 as $t_{2}-t_{1} \rightarrow 0$, which implies that $Q(\Omega)$ is equicontinuous.

Let $B=\overline{\operatorname{co}} Q(\Omega)$. Then it is easy to verify that $Q$ maps $B$ into itself and $B \subset P C(J, E)$ is equicontinuous. Now, we prove that $Q: B \rightarrow B$ is a condensing operator. For any $D \subset B$, by Lemma 7, there exists a countable set $D_{0}=\left\{u_{n}\right\} \subset D$, such that

$$
\beta(Q(D)) \leq 2 \beta\left(Q\left(D_{0}\right)\right)
$$

By the equicontinuity of $B$, we know that $D_{0} \subset B$ is also equicontinuous.

By the fact that

$$
\int_{0}^{a} u(s) d s \in a \overline{\mathrm{co}}\{u(s) \mid s \in J\}, \quad u \in C(J, E),
$$

we have

$$
\begin{aligned}
& \beta\left(\left\{\int_{0}^{t} k(t, s) p_{1}(s, u(s)) d s \mid u \in B, t \in J\right\}\right) \\
& \quad \leq a k_{0} L_{4} \beta(\{u(t) \mid u \in B, t \in J\}), \\
& \beta\left(\left\{\int_{0}^{t} h(t, s) p_{2}(s, u(s)) d s \mid u \in B, t \in J\right\}\right) \\
& \quad \leq a h_{0} L_{5} \beta(\{u(t) \mid u \in B, t \in J\}) .
\end{aligned}
$$

Thus, by (H1), (H2), (39), and Lemma 13, we have

$$
\begin{aligned}
& \beta\left(Q\left(D_{0}\right)(t)\right)=\beta\left(\left\{\mathscr{T}_{\alpha}(t)\left(g\left(u_{n}\right)+u_{0}\right)+\sum_{0<t_{k}<t} \int_{t_{k-1}}^{t_{k}}\left(t_{k}-s\right)^{\alpha-1} \mathcal{S}_{\alpha}(t-s) f\left(s, u_{n}(s),\left(F u_{n}\right)(s),\left(G u_{n}\right)(s)\right) d s\right.\right. \\
& \left.\left.+\int_{t_{k}}^{t}(t-s)^{\alpha-1} \mathcal{S}_{\alpha}(t-s) f\left(s, u_{n}(s),\left(F u_{n}\right)(s),\left(G u_{n}\right)(s)\right) d s+\sum_{0<t_{k}<t} \mathscr{T}_{\alpha}\left(t-t_{k}\right) I_{k}\left(u_{n}\left(t_{k}^{-}\right)\right)\right\}\right) \leq \frac{2 M(m+1)}{\Gamma(\alpha)} \int_{0}^{t}(t \\
& -s)^{\alpha-1} \alpha\left(\mathcal{S}_{\alpha}(t-s) f\left(s, u_{n}(s),\left(F u_{n}\right)(s),\left(G u_{n}\right)(s)\right)\right) d s \leq \frac{2 M(m+1)\left(L_{1}+a k_{0} L_{2} L_{4}+a h_{0} L_{3} L_{5}\right) \tau_{1}^{\alpha}}{\Gamma(\alpha+1)} \beta(D) .
\end{aligned}
$$


Since $Q\left(D_{0}\right) \subset B$ is bounded and equicontinuous, we know from Lemma 6 that

$$
\beta\left(Q\left(D_{0}\right)\right)=\max _{t \in I} \beta\left(Q\left(D_{0}\right)(t)\right) .
$$

Therefore, from (37), (40), and (41), we know that

$$
\begin{aligned}
& \beta(Q(D)) \\
& \leq \frac{2 M(m+1)\left(L_{1}+a k_{0} L_{2} L_{4}+a h_{0} L_{3} L_{5}\right) \tau_{1}^{\alpha}}{\Gamma(\alpha+1)} \\
& \quad \cdot \beta(D) \leq \beta(D) .
\end{aligned}
$$

Thus, $Q: B \rightarrow B$ is a condensing operator. It follows from Lemma 10 that $Q$ has at least one fixed point $u\left(t_{0}\right) \in B$, which is just a solution of problem (4) on the interval $\left[0, \tau_{1}\right]$. This completes the proof.

Corollary 15. Let $E$ be a Banach space. $A: D(A) \subset E \rightarrow E$ be a closed linear operator and $-A$ generates an equicontinuous $C_{0}$-semigroup $T(t)(t \geq 0)$ of uniformly bounded operators in E. Suppose that the conditions (H1)-(H5) are satisfied. Then for every $u_{0} \in P C(J, E)$ there exists a $\tau_{1}=\tau_{1}\left(u_{0}\right), 0<\tau_{1}<$ a such that the nonlocal problem

$$
\begin{aligned}
& { }^{c} D_{t}^{\alpha} u(t)+A u(t)=f(t, u(t),(F u)(t),(G u)(t)), \\
& t \in J, t \neq t_{k}, \\
& \left.\Delta u\right|_{t=t_{k}}=I_{k}\left(u\left(t_{k}^{-}\right)\right), \quad k=1,2, \ldots, m, \\
& u(0)=u_{0}+\sum_{i=1}^{m} c_{i} u\left(t_{i}\right)
\end{aligned}
$$

has a solution $u \in P C\left(\left[0, \tau_{1}\right], E\right)$.

Proof. Let the function $g(u)=\sum_{i=1}^{m} c_{i} u\left(t_{i}\right)$; by the similar way one can easily verify the conditions (H1)-(H5) by properly choosing $c_{i}$. Hence, by Theorem 14, problem (43) has a solution.

Theorem 16. Let $\xi: E \times E \rightarrow R^{+}$be a given function. Assume that the following conditions hold:

(A) there exists $\psi \in \Psi$ such that

$$
\begin{aligned}
& \| f(s, u(s),(F u)(s),(G u)(s)) \\
& \quad-f(s, v(s),(F v)(s),(G v)(s)) \| \leq \frac{\Gamma(\alpha+1)}{3 M(m+1) a^{\alpha}} \\
& \quad \cdot \psi(\|u-v\|), \\
& \|g(u)-g(v)\| \leq \frac{1}{3 M} \psi(\|u-v\|), \\
& \left\|I_{k}\left(u\left(t_{k}^{-}\right)\right)-I_{k}\left(v\left(t_{k}^{-}\right)\right)\right\| \leq \frac{1}{3 M m} \psi(\|u-v\|),
\end{aligned}
$$

for all $t \in J$ and for all $a, b \in E$ with $\xi(a, b) \geq 0$;
(B) there exists $u_{0} \in P C(J, E)$ such that $\xi\left(u_{0}(t), Q u_{0}(t)\right) \geq$ 0 for all $t \in J$, where a mapping $Q: P C(J, E) \rightarrow$ $P C(J, E)$ is defined by

$$
\begin{aligned}
& Q u(t)=\mathscr{T}_{\alpha}(t)\left(g(u)+u_{0}\right)+\sum_{0<t_{k}<t} \int_{t_{k-1}}^{t_{k}}\left(t_{k}-s\right)^{\alpha-1} \\
& \cdot \mathcal{S}_{\alpha}(t-s) f(s, u(s),(F u)(s),(G u)(s)) d s \\
& +\int_{t_{k}}^{t}(t-s)^{\alpha-1} \mathcal{S}_{\alpha}(t-s) f(s, u(s),(F u)(s),(G u) \\
& \cdot(s)) d s+\sum_{0<t_{k}<t} \mathscr{T}_{\alpha}\left(t-t_{k}\right) I_{k}\left(u\left(t_{k}^{-}\right)\right) ;
\end{aligned}
$$

(C) for each $t \in J$, and $u, v \in P C(J, E), \xi(u(t), v(t)) \geq 0$ implies that $\xi(Q u(t), Q v(t)) \geq 0$;

(D) for each $t \in J$, if $\left\{u_{n}\right\}$ is a sequence in $P C(J, E)$ such that $u_{n} \rightarrow u$ in $P C(J, E)$ and $\xi\left(u_{n}(t), u_{n+1}(t)\right) \geq 0$ for all $n \in \mathbb{N}$, then

$$
\xi\left(u_{n}(t), u(t)\right) \geq 0
$$

for all $n \in \mathbb{N}$.

Proof. First of all, let $E=P C(J, E)$. It is easy to see that $u \in E$ is a solution of (4) if and only if $u \in E$ is a solution of the integral equation

$$
\begin{aligned}
& u(t)=\mathscr{T}_{\alpha}(t)\left(g(u)+u_{0}\right)+\sum_{0<t_{k}<t} \int_{t_{k-1}}^{t_{k}}\left(t_{k}-s\right)^{\alpha-1} \\
& \cdot \mathcal{S}_{\alpha}(t-s) f(s, u(s),(F u)(s),(G u)(s)) d s \\
& +\int_{t_{k}}^{t}(t-s)^{\alpha-1} \mathcal{S}_{\alpha}(t-s) f(s, u(s),(F u)(s),(G u) \\
& \cdot(s)) d s+\sum_{0<t_{k}<t} \mathscr{T}_{\alpha}\left(t-t_{k}\right) I_{k}\left(u\left(t_{k}^{-}\right)\right) .
\end{aligned}
$$

Then problem (4) is equivalent to finding $u^{*} \in E$ which is a fixed point of $Q$.

Now, let $u, v \in E$ such that $\xi(u(t), v(t)) \geq 0$ for all $t \in J$. By condition (A), we have

$$
\begin{aligned}
& \|Q u(t)-Q v(t)\|=\| \mathscr{T}_{\alpha}(t)\left(g(u)+u_{0}\right) \\
& +\sum_{0<t_{k}<t} \int_{t_{k-1}}^{t_{k}}\left(t_{k}-s\right)^{\alpha-1} \mathcal{S}_{\alpha}(t-s) \\
& \cdot f(s, u(s),(F u)(s),(G u)(s)) d s+\int_{t_{k}}^{t}(t \\
& -s)^{\alpha-1} \mathcal{S}_{\alpha}(t-s) f(s, u(s),(F u)(s),(G u)(s)) d s \\
& +\sum_{0<t_{k}<t} \mathscr{T}_{\alpha}\left(t-t_{k}\right) I_{k}\left(u\left(t_{k}^{-}\right)\right)-\mathscr{T}_{\alpha}(t)\left(g(v)+v_{0}\right) \\
& -\sum_{0<t_{k}<t} \int_{t_{k-1}}^{t_{k}}\left(t_{k}-s\right)^{\alpha-1} \mathcal{S}_{\alpha}(t-s)
\end{aligned}
$$




$$
\begin{aligned}
& \cdot f(s, v(s),(F v)(s),(G v)(s)) d s-\int_{t_{k}}^{t}(t-s)^{\alpha-1} \\
& \cdot \mathcal{S}_{\alpha}(t-s) f(s, v(s),(F v)(s),(G v)(s)) d s \\
& -\sum_{0<t_{k}<t} \mathscr{T}_{\alpha}\left(t-t_{k}\right) I_{k}\left(v\left(t_{k}^{-}\right)\right)\|\leq M\|(g(u) \\
& -g(v)) \|+\frac{M(m+1)}{\Gamma(\alpha)} \int_{0}^{t}(t-s)^{\alpha-1} \\
& \cdot \| f(s, u(s),(F u)(s),(G u)(s))-f(s, v(s),(F v) \\
& \cdot(s),(G v)(s))\|+M m\| I_{k}\left(u\left(t_{k}^{-}\right)\right)-I_{k}\left(v\left(t_{k}^{-}\right)\right) \| \\
& \leq \frac{1}{3} \psi(\|u-v\|)+\frac{1}{3} \psi(\|u-v\|)+\frac{1}{3} \psi(\|u-v\|) \\
& =\psi(\|u-v\|) .
\end{aligned}
$$

This implies that for each $u, v \in E$ with $\xi(u(t), v(t)) \geq 0$ for all $t \in J$, we obtain that

$$
\|Q x(t)-Q y(t)\| \leq \psi(\|u-v\|)
$$

for all $u, v \in E$. Now, we define the function $\gamma: E \times E \rightarrow$ $[0, \infty)$ by

$$
\gamma(u, v)= \begin{cases}1 & \text { if } \xi(u(t), v(t)) \geq 0 \forall t \in J, \\ 0 \quad \text { otherwise }\end{cases}
$$

and also we define the $w$-distance $p$ on $E$ by $p(u, v)=\|u-v\|$. From (49), we have

$$
\gamma(u, v) p(Q u, Q v) \leq \psi(p(u, v))
$$

for all $u, v \in E$. This implies that $Q$ is an $(\gamma, \psi, p)$-contractive mapping. From condition (B), there exists $u_{0} \in E$ such that $\gamma\left(u_{0}, Q u_{0}\right) \geq 1$. Next, by using condition (C), the following assertions hold for all $u, v \in E$ :

$$
\begin{aligned}
\gamma(u, v) & \geq 1 \Longrightarrow \\
\xi(u(t), v(t)) & \geq 0 \Longrightarrow \\
\xi(Q u(t), Q v(t)) & \geq 0 \Longrightarrow \\
\gamma(Q u, Q v) & \geq 1
\end{aligned}
$$

and hence $Q$ is an $\gamma$-admissible mapping. Finally, from condition (D), we get that condition (iii) of Lemma 11 holds. Therefore, by Lemma 11, we find $x^{*} \in E$ such that $x^{*}=Q x^{*}$ and so $x^{*}$ is a solution of problem (4), which completes the proof.

\section{Applications}

In this section, we present two examples, which illustrate the applicability of our main results.
Throughout this section, we let $E=L^{2}([0, \pi])$ and consider the operator $A: D(A) \subseteq E \rightarrow E$ defined by

$$
\begin{aligned}
D(A) & =\left\{u \in E: \frac{\partial u}{\partial x}, \frac{\partial^{2} u}{\partial x^{2}} \in E, u(0)=u(\pi)=0\right\}, \\
A u & =-\frac{\partial^{2}}{\partial x^{2}} u .
\end{aligned}
$$

It is well known that $-A$ generates a uniformly bounded $C_{0}$ semigroup $(T(t)) t>0$ in $E$.

Example 1. We consider the following impulsive fractional differential equation:

$$
\begin{aligned}
& \frac{\partial^{\alpha}}{\partial t^{\alpha}} u(x, t)-\frac{\partial^{2}}{\partial x^{2}} u(x, t) \\
& =\frac{1}{(t+5)^{2}} \frac{\|u(x, t)\|}{1+\|u(x, t)\|}+\frac{1}{5} \int_{0}^{t} \frac{e^{-(s-t)}}{5} u(x, s) d s \\
& \quad+\frac{1}{5} \int_{0}^{t} \frac{e^{-(s-t) / 2}}{5} u(x, s) d s, \\
& u(0, t)=u(\pi, t), \quad t \geq 0, \\
& \left.\Delta u\right|_{t=1 / 2}=\sin \left(\frac{1}{7}\left\|u\left(x, \frac{1}{2}\right)\right\|\right), \\
& u(x, 0)=u_{0}(x),
\end{aligned}
$$

where $t \in[0,1], x \in(0, \pi), t \neq 1 / 2, \partial^{\alpha} / \partial t^{\alpha}$ is the Caputo fractional-order partial derivative of order $\alpha, 0<\alpha \leq 1, u_{0} \in$ $L^{2}[0, \pi]$. Take $J:=[0,1]$ and so $a=1$.

$$
\begin{aligned}
& \text { Let } \\
& \begin{aligned}
p_{1}(t, u(t, x)) & =p_{2}(t, u(t, x))=u(t, x) \\
F u & =\int_{0}^{t} \frac{e^{-(s-t)}}{5} u(x, s) d s \\
G u & =\int_{0}^{t} \frac{e^{-(s-t) / 2}}{5} u(x, s) d s \\
f(t, u, F u, G u) & =\frac{1}{(t+5)^{2}} \frac{\|u(x, t)\|}{1+\|u(x, t)\|}+F u+G u, \\
I_{k}(u) & =\sin \left(\frac{1}{7}\|u(x, t)\|\right)
\end{aligned}
\end{aligned}
$$

then the impulsive fractional differential equation (54) can be transformed into the abstract form of problem (4).

Next, let $u, v \in C(J, E)$, we calculate

$$
\begin{gathered}
\|F u-F v\|=\| \int_{0}^{t} \frac{e^{-(s-t)}}{5} u(x, s) d s \\
-\int_{0}^{t} \frac{e^{-(s-t)}}{5} v(x, s) d s\left\|\leq \frac{2}{5}\right\| u-v \|, \\
\|G u-G v\|=\| \int_{0}^{t} \frac{e^{-(s-t) / 2}}{5} u(x, s) d s \\
-\int_{0}^{t} \frac{e^{-(s-t) / 2}}{5} v(x, s) d s\left\|\leq \frac{2}{5}\right\| u-v \|,
\end{gathered}
$$




$$
\begin{aligned}
& \|f(t, u, F u, G u)-f(t, v, F v, G v)\| \\
& =\| \frac{1}{(t+5)^{2}}\left(\frac{\|u\|}{1+\|u\|}-\frac{\|v\|}{1+\|v\|}\right)+\frac{2}{25}(F u-F v) \\
& \quad+\frac{2}{25}(G u-G v)\left\|\leq \frac{1}{25}\right\| u-v\left\|+\frac{2}{25}\right\| F u-F v \| \\
& \quad+\frac{2}{25}\|G u-G v\|, \\
& \|f(t, u, F u, G u)\|=\| \frac{1}{(t+5)^{2}} \frac{\|u(x, t)\|}{1+\|u(x, t)\|} \\
& \quad+\frac{1}{5} \int_{0}^{t} \frac{e^{-(s-t)}}{5} u(x, s) d s+\frac{1}{5} \int_{0}^{t} \frac{e^{-(s-t) / 2}}{5} u(x, s) d s \| \\
& \quad \leq \frac{1}{25}\|u\|+\frac{2}{25}\|u\|+\frac{2}{25}\|u\|=\frac{1}{5}\|u\|, \\
& \left\|p_{1}(t, u)-p_{1}(t, v)\right\| \leq 2\|u-v\|, \\
& \left\|p_{2}(t, u)-p_{2}(t, v)\right\| \leq 2\|u-v\|, \\
& \left\|p_{1}(t, u)\right\| \leq 2\|u\|, \\
& \left\|p_{2}(t, u)\right\| \leq 2\|u\| ;
\end{aligned}
$$

hence the condition (H1), (H2) holds with $K_{1}=1 / 25, K_{2}=$ $K_{3}=2 / 25, M_{R}(t)=1 / 5, \Omega(r)=r, b_{1}=b_{2}=2$, and $c_{1}=c_{2}=$ 2. Then we have

$$
\begin{aligned}
\left\|I_{k}(u)-I_{k}(v)\right\| & =\left\|\sin \left(\frac{1}{7} u\right)-\sin \left(\frac{1}{7} v\right)\right\| \\
& \leq \frac{1}{7}\|u-v\|, \\
\left\|I_{k}(u)\right\| & =\left\|\sin \left(\frac{1}{7} u\right)\right\| \leq \frac{1}{7}\|u\| ;
\end{aligned}
$$

for any bounded set $X, Y, Z \in C(J, E), I \subset J$, we have

$$
\begin{aligned}
\beta\left(k(I, I) p_{1}(I, Y)\right) & \leq \frac{2}{5} \beta(Y), \\
\beta\left(h(I, I) p_{1}(I, Z)\right) & \leq \frac{2}{5} \beta(Z), \\
\beta(f(t, X, Y, Z)) & \leq \frac{1}{25} \beta(X)+\frac{2}{25} \beta(Y) \\
& +\frac{2}{25} \beta(Z) .
\end{aligned}
$$

Hence the condition (H4), (H5) holds with $\mu=\rho=1 / 7$, $L_{1}=1 / 25, L_{2}=L_{3}=2 / 25$, and $L_{4}=L_{5}=2 / 5$. Then, by Theorem 14, problem (54) has a solution on $[0,1]$.
Example 2. Consider the following impulsive fractional differential equation:

$$
\begin{aligned}
& \frac{\partial^{\alpha}}{\partial t^{\alpha}} u(x, t)-\frac{\partial^{2}}{\partial x^{2}} u(x, t) \\
& =\frac{t}{25} \frac{\|u(x, t)\|}{1+\|u(x, t)\|}+\frac{1}{5} \int_{0}^{t} \frac{e^{-(s-t)}}{5} u(x, s) d s \\
& +\frac{1}{5} \int_{0}^{t} \frac{e^{-(s-t) / 2}}{5} u(x, s) d s, \\
& u(0, t)=u(\pi, t), \quad t \geq 0, \\
& \left.\Delta u\right|_{t=1 / 2}=\frac{\left|u\left(x,(1 / 2)^{-}\right)\right|}{20+\left|u\left(x,(1 / 2)^{-}\right)\right|}, \\
& u(x, 0)=u_{0}(x)+\sum_{i=1}^{m} \eta_{i} u\left(x, \frac{1}{2}\right),
\end{aligned}
$$

where $t \in[0,1], x \in[0, \pi], t \neq 1 / 2$, and $\partial^{\alpha} / \partial t^{\alpha}$ is the Caputo fractional-order partial derivative of order $\alpha, 0<\alpha \leq 1, u_{0} \in$ $L^{2}[0, \pi], 0<\eta_{1}<\eta_{2}<\cdots<1$, and $\eta_{i}$ is given positive constants with $\sum_{i=1}^{m} \eta_{i}<2 / 15$. Take $J:=[0,1]$ and so $a=1$.

Let

$$
\begin{aligned}
F u & =\int_{0}^{t} \frac{e^{-(s-t)}}{5} u(x, s) d s, \\
G u & =\int_{0}^{t} \frac{e^{-(s-t) / 2}}{5} u(x, s) d s, \\
f(t, u, F u, G u) & =\frac{t}{25} \frac{\|u(x, t)\|}{1+\|u(x, t)\|}+F u+G u, \\
I_{k}(u) & =\frac{\left|u\left(x,(1 / 2)^{-}\right)\right|}{20+\left|u\left(x,(1 / 2)^{-}\right)\right|}, \\
g(u) & =\sum_{i=1}^{m} \eta_{i} u\left(x, \frac{1}{2}\right) ;
\end{aligned}
$$

then the impulsive fractional differential equation (59) can be transformed into the abstract form of problem (43).

Next, letting $u, v \in C(J, E)$, we calculate

$$
\begin{gathered}
\|F u-F v\|=\| \int_{0}^{t} \frac{e^{-(s-t)}}{5} u(x, s) d s \\
-\int_{0}^{t} \frac{e^{-(s-t)}}{5} v(x, s) d s\left\|\leq \frac{2}{5}\right\| u-v \|, \\
\|G u-G v\|=\| \int_{0}^{t} \frac{e^{-(s-t) / 2}}{5} u(x, s) d s \\
-\int_{0}^{t} \frac{e^{-(s-t) / 2}}{5} v(x, s) d s\left\|\leq \frac{2}{5}\right\| u-v \|,
\end{gathered}
$$




$$
\begin{aligned}
& \|f(t, u, F u, G u)-f(t, v, F v, G v)\| \\
& =\| \frac{t}{25}\left(\frac{\|u\|}{1+\|u\|}-\frac{\|v\|}{1+\|v\|}\right)+\frac{2}{25}(F u-F v) \\
& \quad+\frac{2}{25}(G u-G v)\left\|\leq \frac{1}{25}\right\| u-v\left\|+\frac{2}{25}\right\| F u-F v \| \\
& \quad+\frac{2}{25}\|G u-G v\|, \\
& \|f(t, u, F u, G u)\|=\| \frac{t}{25} \frac{\|u(x, t)\|}{1+\|u(x, t)\|} \\
& \quad+\frac{1}{5} \int_{0}^{t} \frac{e^{-(s-t)}}{5} u(x, s) d s+\frac{1}{5} \int_{0}^{t} \frac{e^{-(s-t) / 2}}{5} u(x, s) d s \| \\
& \quad \leq \frac{1}{25}\|u\|+\frac{2}{25}\|u\|+\frac{2}{25}\|u\|=\frac{1}{5}\|u\|, \\
& \left\|p_{1}(t, u)-p_{1}(t, v)\right\| \leq 2\|u-v\|, \\
& \left\|p_{2}(t, u)-p_{2}(t, v)\right\| \leq 2\|u-v\|, \\
& \left\|p_{1}(t, u)\right\| \leq 2\|u\|, \\
& \left\|p_{2}(t, u)\right\| \leq 2\|u\| ;
\end{aligned}
$$

hence the condition (H1), (H2) holds with $K_{1}=1 / 25, K_{2}=$ $K_{3}=2 / 25, M_{R}(t)=1 / 5$, and $\Omega(r)=r$. Then we have

$$
\begin{aligned}
\left\|I_{k}(u)-I_{k}(v)\right\| & =\left\|\frac{u}{20+u}-\frac{v}{20+v}\right\| \leq \frac{1}{20}\|u-v\|, \\
\|g(u)-g(v)\| & =\left\|\sum_{i=1}^{m} \eta_{i} u-\sum_{i=1}^{m} \eta_{i} v\right\| \leq \frac{2}{15}\|u-v\|, \\
\left\|I_{k}(u)\right\| & =\left\|\frac{u}{20+u}\right\| \leq \frac{1}{20}\|u\|, \\
\|g(u)\| & =\left\|\sum_{i=1}^{m} \eta_{i} u\right\| \leq \frac{2}{15}\|u\| ;
\end{aligned}
$$

for any bounded set $X, Y, Z \in C(J, E), I \subset J$, we have

$$
\begin{aligned}
\beta\left(k(I, I) p_{1}(I, Y)\right) & \leq \frac{2}{5} \beta(Y), \\
\beta\left(h(I, I) p_{1}(I, Z)\right) & \leq \frac{2}{5} \beta(Z), \\
\beta(f(t, X, Y, Z)) & \leq \frac{1}{25} \beta(X)+\frac{2}{25} \beta(Y) \\
& +\frac{2}{25} \beta(Z) .
\end{aligned}
$$

Hence the condition (H3), (H4) holds with $\mu=\rho=1 / 7$, $L_{1}=1 / 25, L_{2}=L_{3}=2 / 25, L_{4}=L_{5}=2 / 5$, and $N_{1}=N_{2}=$ $2 / 15$. Therefore, by Corollary 15 , problem (59) has a solution on $[0,1]$.

\section{Conclusions}

In this paper, we studied the existence of solutions for a class of impulsive fractional evolution equations with nonlocal conditions in Banach space by using some fixed point theorems combined with the technique of measure of noncompactness. Our results improve and generalize some known results corresponding to those obtained by others.

\section{Competing Interests}

The authors declare that they have no competing interests.

\section{Acknowledgments}

This work is supported by the National Natural Science Foundation of China (Grant no. 11061031).

\section{References}

[1] R. P. Agarwal, M. Benchohra, and S. Hamani, "A survey on existence results for boundary value problems of nonlinear fractional differential equations and inclusions," Acta Applicandae Mathematicae, vol. 109, no. 3, pp. 973-1033, 2010.

[2] M. Benchohra, J. R. Graef, and S. Hamani, "Existence results of nonlinear fractional differential equations on reflexive Banach spaces," Electronic Journal of Qualitative Theory of Differential Equations, vol. 54, pp. 1-10, 2010.

[3] M. Benchohra, S. Hamani, and S. K. Ntouyas, "Boundary value problems for differential equations with fractional order and nonlocal conditions," Nonlinear Analysis: Theory, Methods \& Applications, vol. 71, no. 7-8, pp. 2391-2396, 2009.

[4] X. B. Shu and Q. Q. Wang, "The existence and uniqueness of mild solutions for fractional differential equations with nonlocal conditions of order $1<\alpha<2$," Computers and Mathematics with Applications, vol. 64, pp. 2100-2110, 2012.

[5] M. Benchohra, J. Henderson, and S. Ntouyas, "Impulsive differential equations and inclusions," Contemporary Mathematics and its Applications, vol. 2, 2006.

[6] V. Lakshmikantham, D. D. Bainov, and P. S. Simeonov, Theory of Impulsive Differential Equations, vol. 6, World Scientific Publishing, Singapore, 1989.

[7] Y. X. Li, "Existence of solutions to initial value problems for abstract semilinear evolution equations," Acta Mathematica Sinica, vol. 48, no. 6, pp. 1089-1094, 2005.

[8] Z. M. He and X. M. He, "Monotone iterative technique for impulsive integro-differential equations with periodic boundary conditions," Computers \& Mathematics with Applications, vol. 48, no. 1-2, pp. 73-84, 2004.

[9] L. Byszewski, "Theorems about the existence and uniqueness of solutions of a semilinear evolution nonlocal Cauchy problem," Journal of Mathematical Analysis and Applications, vol. 162, no. 2, pp. 494-505, 1991.

[10] G. M. N’Guérékata, “A Cauchy problem for some fractional abstract differential equation with non local conditions," Nonlinear Analysis: Theory, Methods \& Applications, vol. 70, no. 5, pp. 1873-1876, 2009.

[11] K. Balachandran and J. Y. Park, "Nonlocal Cauchy problem for abstract fractional semilinear evolution equations," Nonlinear Analysis: Theory, Methods \& Applications, vol. 71, no. 10, pp. 4471-4475, 2009. 
[12] K. Balachandran, S. Kiruthika, and J. J. Trujillo, "Existence results for fractional impulsive integrodifferential equations in Banach spaces," Communications in Nonlinear Science and Numerical Simulation, vol. 16, no. 4, pp. 1970-1977, 2011.

[13] H. D. Gou and B. L. Li, "Local and global existence of mild solution to impulsive fractional semilinear integro-differential equation with noncompact semigroup," Communications in Nonlinear Science and Numerical Simulation, vol. 42, pp. 204214, 2017.

[14] Y. Zhou and F. Jiao, "Existence of mild solutions for fractional neutral evolution equations," Computers \& Mathematics with Applications, vol. 59, no. 3, pp. 1063-1077, 2010.

[15] P. Chen and Y. Li, "Existence of mild solutions for fractional evolution equations with mixed monotone nonlocal conditions," Zeitschrift für Angewandte Mathematik und Physik, vol. 65, no. 4, pp. 711-728, 2014.

[16] J. Banas and K. Goebel, Measure of Noncompactness in Banach Spaces, vol. 60 of Lecture Notes in Pure and Applied Mathematics, Marcel Pekker, New York, NY, USA, 1980.

[17] M. M. El-Borai, "Some probability densities and fundamental solutions of fractional evolution equations," Chaos, Solitons and Fractals, vol. 14, no. 3, pp. 433-440, 2002.

[18] H. Lakzian, D. Gopal, and W. Sintunavarat, "New fixed point results for mappings of contractive type with an application to nonlinear fractional differential equations," Journal of Fixed Point Theory and Applications, vol. 18, no. 2, pp. 251-266, 2016.

[19] K. Deimling, Nonlinear Functional Analysis, Springer, New York, NY, USA, 1985. 


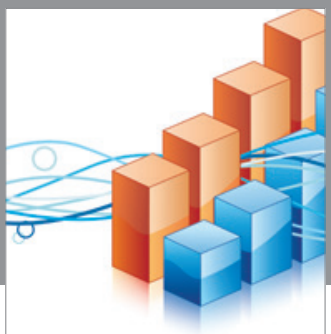

Advances in

Operations Research

vatem alat4

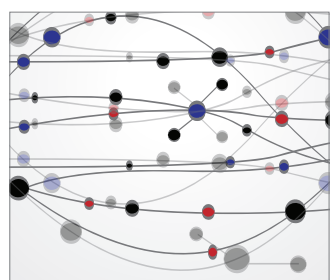

\section{The Scientific} World Journal
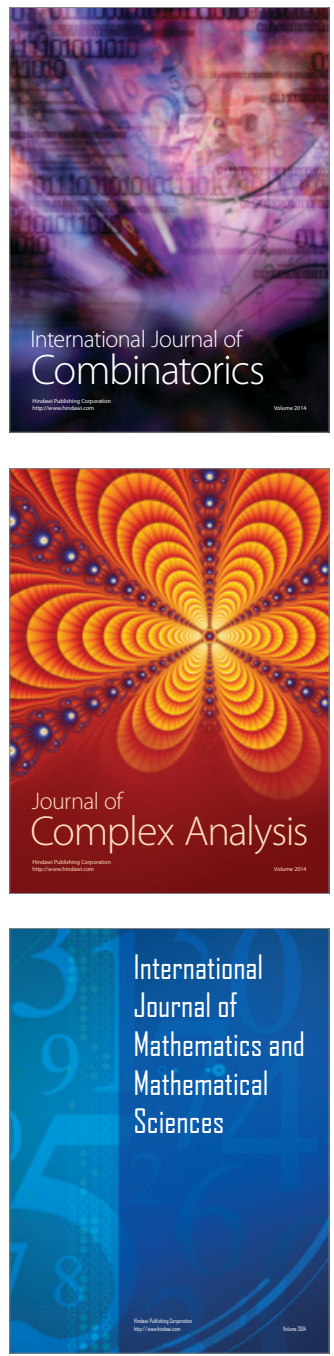
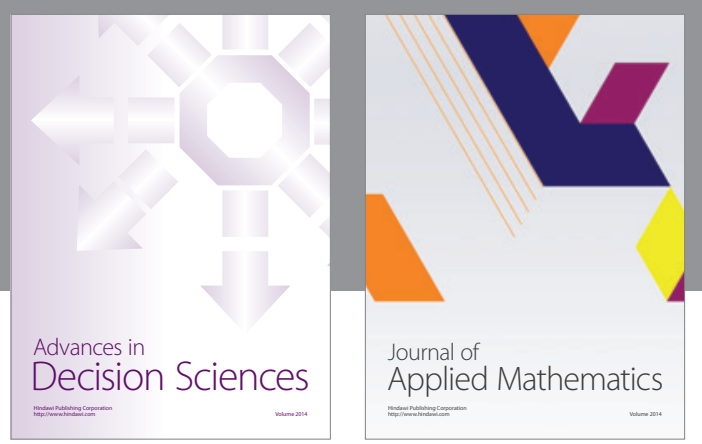

Algebra

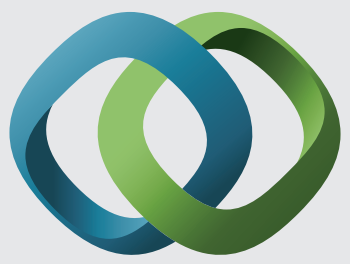

\section{Hindawi}

Submit your manuscripts at

http://www.hindawi.com
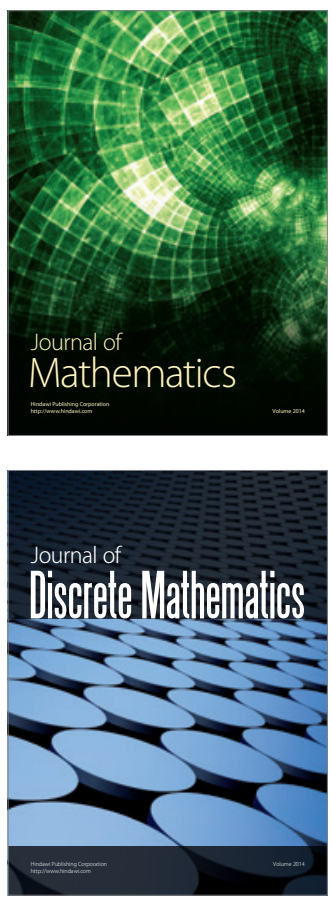

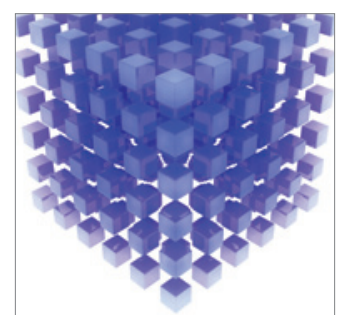

Mathematical Problems in Engineering
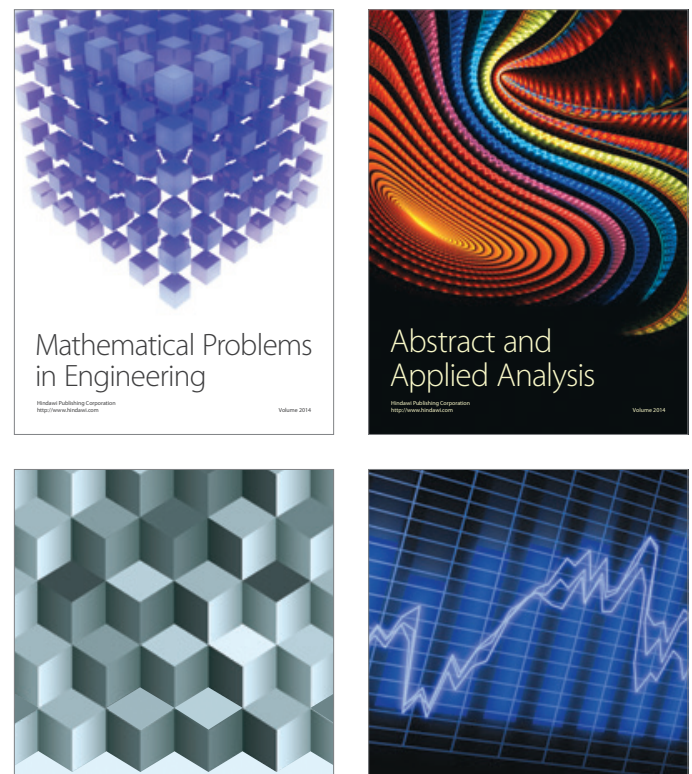

Journal of

Function Spaces

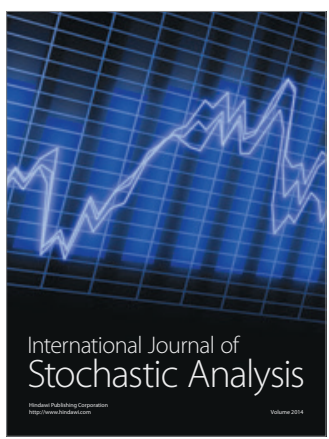

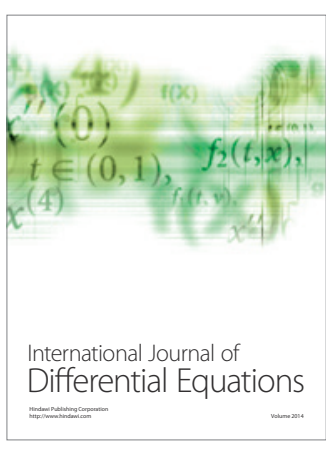
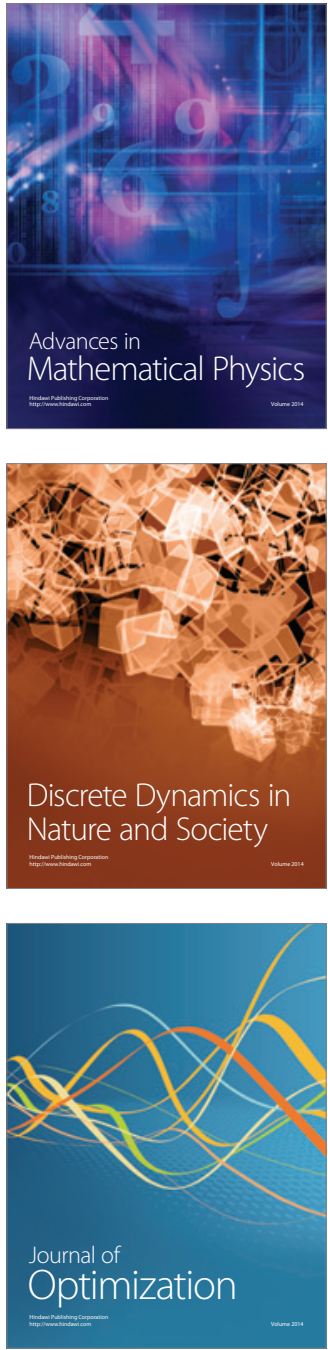